\title{
Manipulation of Ceramic Fibers to EXpressLO'M Grids for FIB/TEM Analysis
}

Lucille A. Giannuzzi ${ }^{1,2}$, Shay L. Harrison ${ }^{3}$, Kirk L. Williams ${ }^{3}$, Ram K. Goduguchinta ${ }^{3}$, Erik G. Vaaler ${ }^{3}$, John L. Schneiter ${ }^{3}$, and Joseph Pegna ${ }^{3}$

${ }^{1 .}$ L.A. Giannuzzi \& Associates LLC, Fort Myers, FL 33971 USA.

${ }^{2}$ EXpressLO LLC, Lehigh Acres, FL 33971 USA.

${ }^{3}$.Free Form Fibers, Saratoga Springs, NY 12866 USA.

Focused ion beam (FIB) lift out techniques for transmission electron microscope (TEM) can be used for fibers, powders, and particulates [1]. In addition, samples can be pre-thinned and glued to half grids for subsequent FIB milling along the length of the sample [2]. Particles can be manipulated and glued to a half grid for subsequent FIB/TEM using an ex situ lift out (EXLO) micromanipulator system [1,3]. In this paper we describe use of an EXLO micromanipulator system to pick up ceramic fibers using adhesion forces and manipulate them across an open slotted EXpressLO ${ }^{\mathrm{TM}}$ grid carrier [4]. M-Bond glue is used to adhere the sample to the grid in a manner similar to that described in [1] and elsewhere in this volume $[3,5]$. This Pick\&Place ${ }^{\mathrm{TM}}$ method is used to manipulate fibers for both longitudinal and transverse cross section analysis.

Figures 1 and 2 show light optical micrographs of the Pick\&Place ${ }^{\mathrm{TM}}$ process for fiber specimen preparation. The probe picks up a $30 \mu \mathrm{m}$ diameter, $400 \mu \mathrm{m}$ long fiber in figure 1a. The fiber is manipulated to an EXpressLO ${ }^{\mathrm{TM}}$ grid previously painted with $\mathrm{M}$-Bond adhesive in figure $1 \mathrm{~b}$ resulting in a fiber ready for longitudinal FIB milling in figure 1c. Note that the $400 \mu \mathrm{m}$ long fiber extends along 4 open slots of the grid where FIB milling is possible. A piece of fiber was fractured for transverse preparation and the probe nears it in figure $2 \mathrm{a}$. The probe positions the fiber piece for pick up in figure $2 \mathrm{~b}$ and is manipulated to an EXpressLO ${ }^{\mathrm{TM}}$ grid previously prepared with $\mathrm{M}$-Bond glue shown in figure $2 \mathrm{c}$. Figure 3 shows scanning electron microscopy (SEM) images of the (a) longitudinal and (b) transverse cross sectioned FIB milled fiber. Note that the fiber fracture is evident in figure 3b. Figure 4 shows bright field (BF) TEM images of the (a) longitudinal and (b) transverse cross sectioned FIB milled fiber. Movies detailing the manipulation process may be viewed in reference [6]. This Pick\&Place ${ }^{\mathrm{TM}}$ manipulation technique combined with FIB milling for TEM specimen preparation allows for easy and site specific cross sectioning of ceramic fibers in both longitudinal and transverse orientations. The manipulation process is fast and easy and does not require additional expensive FIB time needed for lift outs [7].

References:

[1] L.A. Giannuzzi et al., in L.A. Giannuzzi and F.A. Stevie (eds.) Intro. to Focused Ion Beams, (2005) Springer, p 201-228.

[2] R. Anderson and S.J. Klepeis, in L.A. Giannuzzi and F.A. Stevie (eds.) Intro. to Focused Ion Beams, (2005) Springer, 173-200.

[3] S. Vitale et al., Microsc. Microanal., 22 (2016) (this volume).

[4] L.A. Giannuzzi et al., Microsc. Microanal., 21 (2015) p.1032.

[5] P.A. Anzalone and L.A. Giannuzzi, Microsc. Microanal., 22 (2016) (this volume).

[6] www.YouTube.com/LAGiannuzzi/videos

[7] FIB work was performed at the Arizona State University John M. Cowley Center for High Resolution Electron Microscopy, Tempe, AZ, and courtesy of Technical Sales Solutions, Beaverton, OR. TEM images were obtained using an FEI TF20 at Portland State University. 


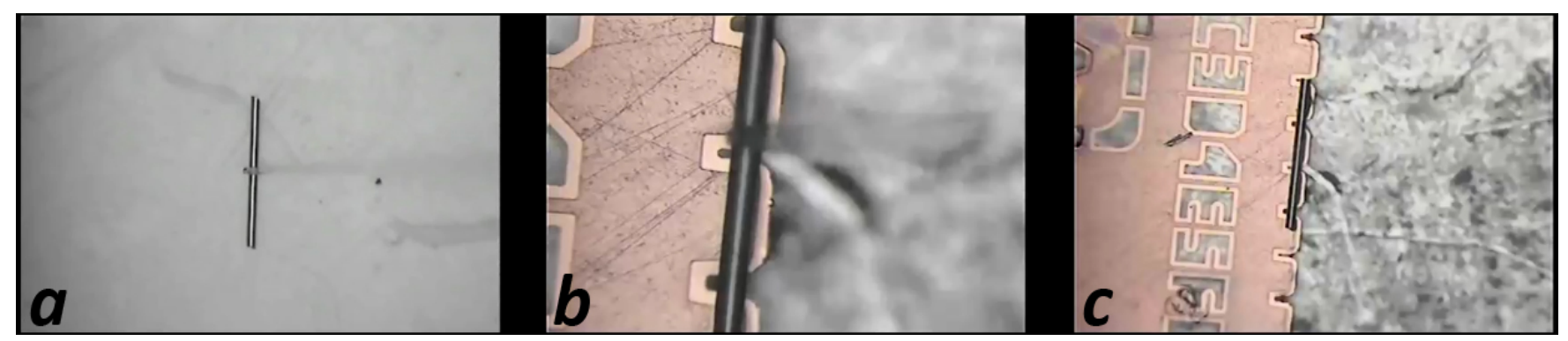

Figure 1. Light optical micrographs of the Pick\&Place ${ }^{\mathrm{TM}}$ manipulation process for longitudinal cross section of $400 \mu \mathrm{m}$ long ceramic fiber. (a) The glass probe picks up the fiber. (b,c) The fiber is manipulated onto the EXpressLO ${ }^{\mathrm{TM}}$ grid previously painted with glue.

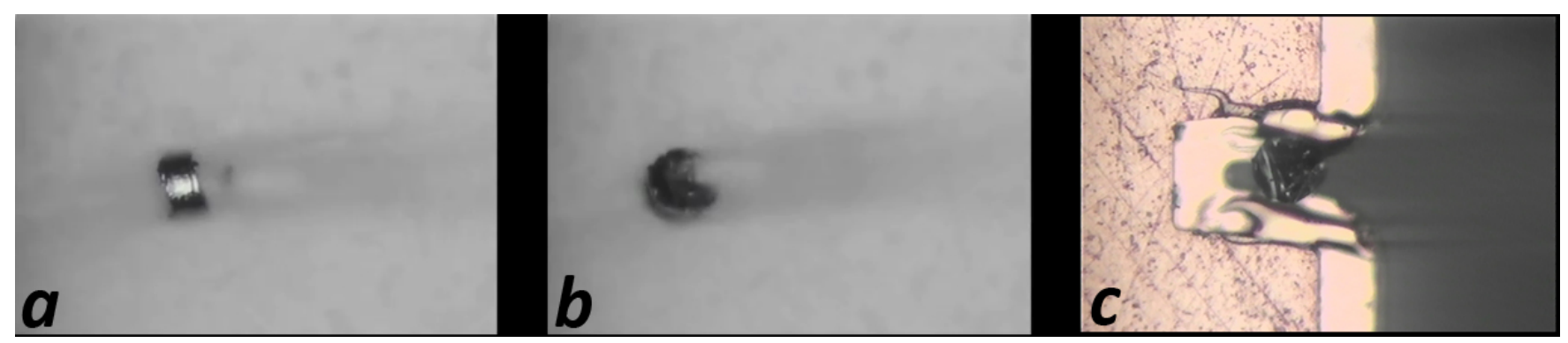

Figure 2. Light optical micrographs of the Pick\&Place ${ }^{\mathrm{TM}}$ manipulation process for transverse cross section of a $30 \mu \mathrm{m}$ diameter ceramic fiber. (a) The glass probe approaches a piece of fiber. (b) The glass probe flips the fiber section over and picks it up. (c) The fiber section is positioned onto the EXpressLO ${ }^{\mathrm{TM}}$ grid previously painted with glue.

(a)

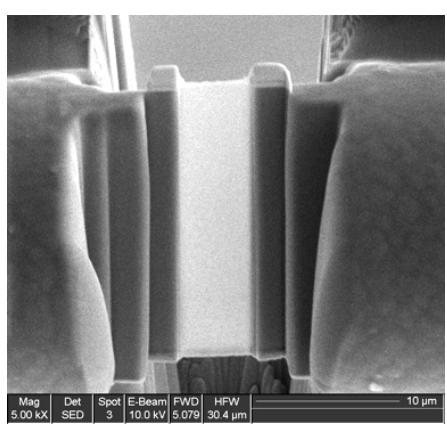

(b)

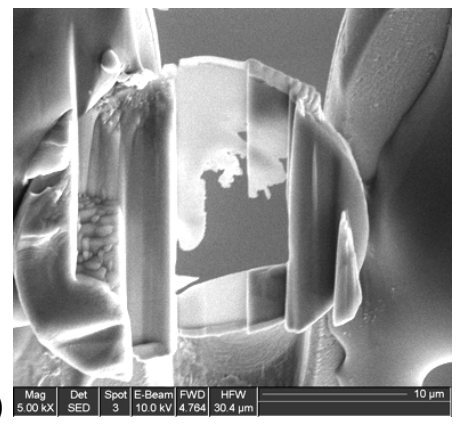

Figure 3. SEM images of the (a) longitudinal and (b) transverse cross sectioned FIB milled fiber.

(a)

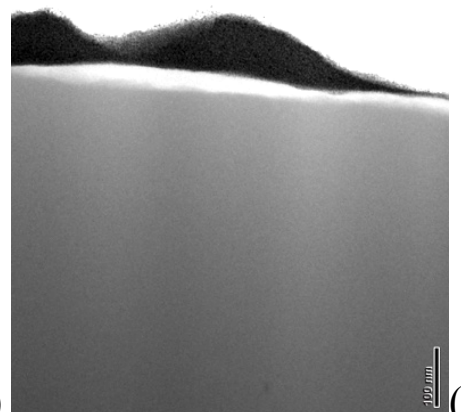

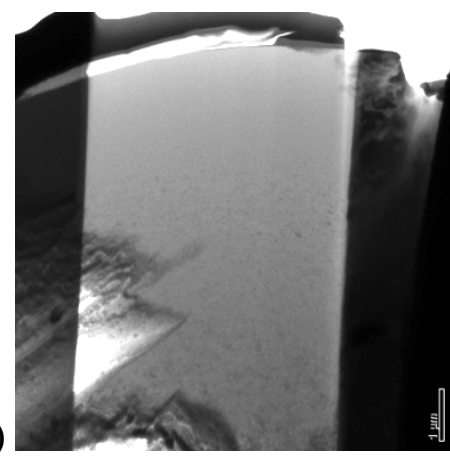

Figure 4. $200 \mathrm{keV}$ BFTEM images of the (a) longitudinal and (b) transverse cross sectioned fiber. 